\title{
Classification and diagnosis of syncope
}

\section{Introduction and definitions}

Syncope is defined as a transient loss of consciousness secondary to cerebral hypoperfusion, characterized by a short duration (usually less than one minute), rapid onset and spontaneous resolution. Adequate diagnosis and classification of syncope may help stratify the risk and define treatment options, leading to better outcomes. However, etiological names may vary and generalized accepted terms are necessary when referring to specific causes of syncope. Among the causes of syncope, there are three main etiologies. The first cause is reflex syncope, frequently known as neurally mediated. The mechanism behind reflex syncope involves cardioinhibition (excessive parasympathetic tone) and vasodepression (decreased sympathetic output) Reflex syncope is defined as a disorder of the autonomic regulations of postural tine, leading to loss of consciousness, bradycardia and hypotension. ${ }^{1}$ Reflex syncope can be divided into vasovagal, situational and carotid sinus syndrome. The first cause is vasovagal syncope is defined as a syncope secondary to an exaggerated parasympathetic response, usually leading to vagal symptoms prior or after the event. ${ }^{2}$ Vasovagal syncope is also divided in positional and emotional. The second cause is situational syncope, defined as the subtype of vasovagal syncope triggered by physiologic stimuli such as micturition, gastrointestinal stimulation, cough or exercise. ${ }^{2}$ The third cause is carotid sinus syndrome leading to syncope, defined as a response to carotid sinus massage that includes reproduction of spontaneous symptoms. ${ }^{2}$ The second etiology is orthostatic hypotension induced syncope. This is defined as a drop higher than $20 \mathrm{mmHg}$ when changing from supine position to stating position. This etiology is mostly secondary to medications. Other causes include primary and secondary autonomic dysfunction (neurogenic syncope) and volume depletion.

The most common medications implied in drug induced orthostatic hypotension are anti-hypertensive medications (diuretics, vasodilators). Primary autonomic dysfunction may be secondary to dementias (particularly Lewy body dementia), Parkinson disease and pure autonomic failure. Secondary causes include most commonly diabetes, amyloidosis, autoimmune neuropathies and trauma induced neuropathy. ${ }^{3}$ Finally, the third cause is cardiac syncope. Cardiac syncope is defined as a syncope secondary to a cardiac pathology. This is probably the most unusual presentation of syncope, but certainly the one that primary care physicians must always rule out. Cardiac causes include arrhythmias, structural heart disease and cardiopulmonary causes. Arrhythmias are the most common cause of cardiac syncope. They are secondary to bradyarrhytmias (sinus, atrioventricular and bundle of Hiss conduction involvement) and tachyarrhythmias. Structural causes include valvulopathies (aortic stenosis), ischemia, cardiac masses, abnormal coronary arteries and hypertrophic cardiomyopathy. Cardiopulmonary causes include pulmonary hypertension or pulmonary emboli.

\section{Diagnostic approach}

The most important diagnostic approach for syncope is performing a complete physical examination, in which the number, frequency and duration of the syncopal episodes is assessed. Also, determining the

\author{
Volume 2 Issue 3 - 2018 \\ Juan Simon Rico-Mesa,' Maria Antonia Rico- \\ Mesa \\ 'Department of Cardiovascular Diseases, Mayo Clinic, USA \\ ${ }^{2}$ Department of Medicine, CES University, Colombia
}

\begin{abstract}
Correspondence: Juan Simon Rico-Mesa, Department of Cardiovascular Diseases, Mayo Clinic, Rochester, MN, USA, Tel
\end{abstract} 50728425 I ,Email juansimonrico@hotmail.com

Received: May 28, 2018 | Published: June 21, 2018

onset would help clarify the etiology. Determining the position in which the syncope occurs is also important: syncopal episodes in the erect position are most related to orthostatic etiology, in comparison to episodes occurring in the supine position, most likely related to cardiac causes such as arrhythmias. ${ }^{1}$ In addition, determining the preceding and following symptoms related to the syncopal are extremely useful. Finally, establishing past medical history, family history and medications would enable the physician to guide his clinical judgment. These last three components are particularly important because they may relate to cardiac causes if found positive; Medical history including neurological conditions, psychiatric illnesses and heart disease. Medications are broad, including diuretics and antihypertensive, leading to low cerebral blood flow. Moreover, medications prolonging the QT interval may induce antiarrhythmic episodes. These medications include but not limited to antipsychotic medications, certain antidepressants and antibiotics (fluoroquinolones, azoles and macrolides). After obtaining a complete medical history, performing a physical examination is mandatory. Specifically, assessing the vital signs, the orthostatic maneuvers, the cardiac findings and the neurological examination as they help establish the cause and determine if it is malignant. ${ }^{4}$

Finally, all patients with suspected or confirmed syncope should undergo an electrocardiogram, as a cost-effective screening exam for cardiac etiologies. In cases in which an abnormal electrocardiographic pattern is suspected, a cardiologist should be consulted. The cardiologist should define if the patient is a suitable candidate for ambulatory electrocardiographic monitoring (Holter monitoring, loop recorder devices), exercise testing, carotid sinus massage or electrophysiology studies. When additional cardiac abnormalities such as structural heart disease or pulmonary embolism/hypertension are suspected, or known, an echocardiography should be pursued. ${ }^{5}$ Neurologic testing and tilt table testing may be needed if reflex syncope is suspected. ${ }^{1}$ Tilt table testing consist of detecting changes in the heart rate and blood pressure mediated by the autonomous nervous system. The test consists of two parts: the first one works by inducing periodical changes in the position of a patient lying down in a table. After tilting the table, changes in these cardiac parameters are evaluated. The second phase consists of evaluating these exact cardiac 
parameters after administering medications. Testing After completing these important steps in the physical examination, the physician must stratify the patient based on its risk of developing complications and therefore, determining if he is suitable for further testing or treatment. Risk categories are subjective and guidelines haven't establish a clear consensus, however, there are clear high risk criteria that should raise extreme awareness on the physician. High risk criteria include syncope during exertion, syncope in supine position, anemia, electrolyte disturbances, family history of sudden cardiac death, electrocardiographic abnormalities such as atrioventricular reentrant tachycardia, Brugada pattern/syndrome, QRS prolongation, QT alterations, congenital cardiac abnormalities such as arrhytmogenic right ventricle cardiomyopathy and any other cardiac illness that guarantee the use of medications or device therapy interventions. ${ }^{6}$ Moderate risk syncope is a gray area, in which the treatment and further studies are made in a case by case basis. Most of the times, patients with a past medical history of structural heart disease without any findings of active cardiac illness are included in this group. On the other hand, low risk patients include those in whom cardiac causes were ruled out or not suspected. This last group includes mostly patients with suspected reflex syncope and orthostatic syncope.

\section{Conclusion}

Obtaining an accurate diagnosis of syncope is essential for the guidance of further treatment. Once a clear diagnosis is obtained, risk stratification is performed. This guarantees a cost-effective approach while providing optimal treatment. Syncope definitions tend to vary among textbooks, articles, guidelines and consensus. Despite that, the primary care physician should adhere to the most updated guidelines accepted by his medical society, so terms can be generalized and common mistakes related to definition confusion can be avoided.

\section{Acknowledgements}

None.

\section{Conflict of interest}

The author declares there is no conflict of interest.

\section{References}

1. Hauk L. Syncope Evaluation and Treatment Guidelines from ACC, AHA, and HRS. Am Fam Physician. 2018;97(7):478-479.

2. Sutton R. Reflex syncope: Diagnosis and treatment. J Arrhythmia. 2017;33(6):545-552.

3. Palma J-A, Kaufmann H. Epidemiology, Diagnosis, and Management of Neurogenic Orthostatic Hypotension. Mov Disord Clin Pract. 2017;4(3):298-308.

4. Gehi AK, Narla V. Syncope: Laying the Groundwork for a Path Forward. JACC Clin Electrophysiol. 2018;4(2):274-276.

5. Ungar A, Rafanelli M. Syncope: Electrocardiographic and Clinical Correlation. Card Electrophysiol Clin. 2018;10(2):371-386.

6. Thiruganasambandamoorthy V, Stiell IG, Sivilotti ML, et al. Risk stratification of adult emergency department syncope patients to predict short-term serious outcomes after discharge (RiSEDS) study. BMC Emerg Med. 2014;14;14:8.

7. Berecki-Gisolf J, Sheldon A, Wieling W, et al. Identifying Cardiac Syncope Based on Clinical History: A Literature-Based Model Tested in Four Independent Datasets. PLoS ONE. 2013;24;8(9):e75255. 